\title{
Prevalence of Mycoplasma pneumoniae, Chlamydia pneumoniae and Legionella pneumophila in LRTI Patients in a Tertiary Care Center, Karimnagar
}

\author{
G. Sowjanya ${ }^{1}$, Amar C. Sajjan ${ }^{2}$, G. Swetha ${ }^{3}$, B. Archana ${ }^{4}$ and Aparna ${ }^{5}$ \\ Department of Microbiology, Chalmeda Anand Rao institute of Medical sciences (CAIMS), \\ Karim nagar, Telanagana, India \\ *Corresponding author
}

\begin{abstract}
Keywords
Atypical

pneumonia, $\operatorname{IgM}$, ELISA

Article Info

Accepted:

15 April 2019

Available Online:

10 May 2019 pneumoniae, Chlamydophila pneumoniae, and Coxiella burnetti" and Viruses. Mycoplasma pneumoniae is the most important and common cause of communityacquired pneumonia (CAP). The conventional detection methods (culture) lack sensitivity and takes longer duration of time for detection. The study was undertaken during November 2018to April 2019, 102 patients with respiratory tract infections were enrolled into this study. M. pneumoniae, Chlamydia pneumoniae and Legionella pneumophila from respiratory tract infections was detected by enzyme-linked immunosorbent assay (ELISA). Atypical bacteria isolated from pneumonia patients are Chlamydia pneumoniae in 5(4.9\%), Mycoplasma pneumoniae7 (6.8\%), and Legionella pneumophila15 (14.7\%) of patients detected by ELISA. Atypical pneumonia infection was mostly prevalent in patients 16-76 years old. Most infections (90\%) were community acquired and cough, fever, dyspnea, and malaise were among the most common symptoms. Pneumonia due to the Atypical bacterial infection is infrequent in Chalmeda Anand Rao institute of Medical sciences and the clinical symptoms of the patients were determined to be mild. The ELISA method also proved to be more sensitive and reliable than culture assays in the detection of $M$. pneumoniae.
\end{abstract}

A B S T R A C T

"Atypical pneumonia" has been defined as "any Community acquired pneumonia (CAP) that is different to that caused by Streptococcus pneumoniae", to indicate "a CAP caused by one of several identified pathogens including Legionella pneumophilia, Mycoplasma

\section{Introduction}

The atypical respiratory pathogens Mycoplasma pneumoniae, Chlamydia pneumoniae and Legionella pneumophila are now recognised as a significant cause of acute respiratory-tract infections, but they remain colorless after Gram-staining and are difficult to identify by conventional bacterial culture tests. It is reported that patients with atypical pneumonia were more likely to have normal or reduced white blood cell counts.(Huang $\mathrm{HH}, 2003)$ However some published data showed that between atypical pneumonia and general bacterial pneumonia, there were no significant differences in the symptoms such 
as fever, cough, productive sputum, and the sign of lung rales (Porath, 1997 and Monsieur, 1997).

Also, X-ray findings and the increase of white blood cell counts and percentage of neutrophils were similar between them. Especially, patients with pneumonia caused by L. pneumophila presented with the typical symptoms of Streptococcus pneumonia while patients with $\mathrm{S}$. pneumonia also presented with the symptoms of atypical pneumonia (Yu, 2003). However, beta-lactams are not effective for atypical pneumonia. Therefore, laboratory detection methods and clinical biology research on the diagnosis and treatment of atypical pathogens infection is particularly important.

\section{Atypical pathogens}

Atypical organisms such as $M$. pneumoniae, C. pneumoniae, and L. pneumophila are implicated in cases of communityacquired pneumonia (CAP). M. pneumoniae in 1962 was successfully isolated (Chanock, 1962). M. pneumoniae lacks a cell wall, but it can grow in artificial culture medium, and it is the most frequent pathogens found in patients with CAP. C. pneumoniae found in 1986 is intracellular organisms and is a common cause of acute and chronic respiratory tract infections (Grayston, 1990). Legionella was found in 1976 because it caused infection outbreak of America veterans. It has been confirmed more than 50 Legionella species, a total of 70 serotypes. L. pneumophila with 16 serotypes is intracellular organisms and closely related to human infection (Diederen, 2008).

\section{Atypical pathogens in community acquired pneumonia}

With the widespread use of antibiotics, the change of living environment and constantly updated diagnostic methods, we found atypical pathogen played an important role in CAP. A prospective study was performed on 665 consecutive adult patients with CAP at 12 centers in 7 Chinese cities between 2003 and 2004 (Liu, 2006). The results showed that atypical pathogens caused $32.4 \%$ cases of CAP, of which $20.7 \%$ M. pneumoniae, $6.6 \%$ C. pneumoniae and $5.1 \%$ L. pneumophila. Of 195 patients with a bacterial pathogen, an atypical pathogen was identified in $10.2 \%$ cases. Survey of Cao et al. on the etiology and clinical outcomes of CAP treated in an ambulatory setting showed that the most common pathogens were $M$. pneumoniae $(29.4 \%)$ and then virus copathogens $(2.5 \%)$ (Cao, 2010). The previous research data of "pathogens monitoring network among adults with CAP in Beijing" showed that of 410 patients with $\mathrm{CAP}, \geq 4$-fold increase of paired serum $M$. pneumoniae IgG antibody titer was observed in $18.8 \%$ of cases. (Qu, 2012). Atypical pathogens, rather than $\mathrm{S}$. pneumoniae, become the most important pathogen of adult CAP.

\section{Materials and Methods}

The study was conducted during the period November 2018 to April 2019. The study group consists of 100 patients attending medical outpatient department and admitted in Chalmeda Anand Rao Institute of Medical sciences, Karimnagar.

\section{Inclusion criteria}

Patients with Community Acquired Pneumonia.

Acute exacerbation of Bronchial asthma

Chronic Obstructive Pulmonary Disease

Age 16 to 65 years and

Patients presenting with symptoms and signs of fever, cough, dysponea and headache.

Presence of new pulmonary infiltrates on chest $\mathrm{x}$ ray. 


\section{Exclusion criteria}

Patients on Antibiotics

Hospital Acquired Pneumonia

Pulmonary Tuberculosis

Bronchiectasis

Interstitial lung disease

Age $<16$ years and $>65$ years

\section{Sample collection and processing}

Blood collection: The skin over the vein was cleaned with $70 \%$ alcohol and allowed to dry. Then povidone iodine was applied was allowed to dry for 1 minute. Blood sample was collected into a sterile bottle without anticoagulant. It was allowed to stand for formation of clot. Then it was centrifuged and supernatant was taken and stored at $-20^{\circ \mathrm{C}}$.

\section{Processing}

All samples were subjected to ELISA to detect IgM antibodies for Mycoplasma pneumoniae, Chlamydia pneumoniae and Legionella pneumophila.

Test was performed according to manufacturer's instructions:

(EUROIMMUN Anti-Mycoplasma pneumoniae ELISA (IgM),

EUROIMMUN Anti-Chlamydia pneumonia ELISA (IgM),

EUROIMMUN Anti-Legionella pneumophila ELISA (IgM)

EUROIMMUN recommends interpreting results as follows:

Ratio <0.8: negative

Ratio $>0.8$ : to $<1.1$ : borderline

Ratio >1.1: positive

\section{Results and Discussion}

Seroprevalence was detected by IgM antibody test using ELISA(EUROIMMUNE) microplate wells coated with antigens (detergent extract of Mycoplasma pneumoniae, strain MAC ATCC 15531, MOMP of CWL-029 strain of Chlamydia pneumoniae, LPS Legionella pneumophila strain 1-7) for IgM detection of Mycoplasma pneumonia, Chlamydia pneumoniae and Legionell pneumophila.

Of the 102 patients studied over the 6-month study period, IgM ELISA revealed 5(4.9\%) chlamydia pneumonia, $7(6.8 \%)$ for Mycoplasma pneumoniae and 15 (14.7\%) legionella pneumophila positive cases, respectively. The highest prevalence rate of M. pneumoniae infection was diagnosed in patients of Age16-76 years old.

The present study detected IgM antibodies for Mycoplasma pneumoniae in $6.8 \%$ patients. According to study by Rama Chaudhry et al., 2013 India IgM antibodies for Mycoplasma pneumoniae detected in $4.47 \%$ of patients of Community acquired pneumonia (Rama Chaudhry et al., 2013).

According to a study by Liu Fang Ching et al., (2008) IgM antibodies for mycoplasma pneumonia were detected in $3.7 \%$ in adults. In year 2004 and 2.9\% in year 2005 (Liu Fang Ching, 2008).

Present study shows similarity in prevalence to these two studies. In the present study IgM antibodies for $M$. pneumoniae has been noted in 5 cases of CAP and 2 cases of Bronchial asthma with acute exacerbations.

Blasi et al., (2004) Europe: reported a role for C. pneumoniae and M. pneumoniae infection as a trigger for 5-30\% episodes of wheezing or acute asthma exacerbations (Blasi, 2004). 
Paraskevi Xepapadaki et al., (2008): M. pneumoniae was associated with hospitalization for asthma exacerbation $18 \%$.

Rama Chaudhry et al., (2013) found that males are more commonly affected than females Male to female ratio is 1:6.

The present study showing Out of 7 cases positive for $M$. pneumoniae $4(57 \%)$ cases were males and $3(42 \%)$ female.

Our study detected IgM antibodies for Legionella pneumophila in $14.7 \%$ of patients.

According to study by Ewing et al., (2002) in Europe: present data on a large population, hospitalized with acute exacerbations of COPD, which provides evidence for the first time for Legionella spp. infection as a potential underlying pathogen in as many as $16.7 \%$ of cases detected by serology (Ewing, 2002).

According to study by Sabah Javed et al., (2010) IgM antibodies for Legionella pneumophila detected in $15.92 \%$ of cases. most common presenting symptoms are fever $80.6 \%$,cough $96.7 \%$,dyspnea $58 \%$,headache $16.1 \%$ (Sabah Javed, 2010).

The patients included in our study showing fever $(76.6 \%) 69$, cough $(87.7 \%) 72$, dyspnea
(38.8\%) 35, head ache (8.8\%) 8. Almudenarojas et al., (2005) reported 29.7\% positive for IgM $L$. pneumophila and that most cases of legionellosis are caused by serogroup-1 (Almudenarojas, 2005).

Direct methods of diagnosis include culturing, direct fluorescent staining, and antigen detection in urine. While the first two methods display low and variable sensitivities. The latter has become a reference technique in most laboratories, enabling easy and early diagnosis of legionellosis. Indirect immune fluorescence is the most common method for serological diagnosis. Although serology yields good sensitivity and specificity data. The delay in the development of a measurable antibody response constitutes a major drawback for diagnosis in the acute patient. Immunoglobulin M ( $\operatorname{IgM})$ detection is widely used in infectious serology, since IgM appears earlier in the course of a disease; however, despite its reported validity for the diagnosis of legionellosis, its use is not widespread and some authors consider it of limited value. The Enzyme-linked immunosorbentassay (ELISA) technique, which generally shows higher sensitivity and better characteristics in terms of both automation and objective measurement than immuno fluorescence (Table 1).

Table.1 Ig M antibodies detection of atypical pathogen by ELISA (No=102)

\begin{tabular}{|l|l|l|}
\hline Pathogen & No & $\%$ \\
\hline Chlamydia pneumoniae & 5 & 4.9 \\
\hline Mycoplasma pneumoniae & 7 & 6.8 \\
\hline Legionella pneumonia & 15 & 14.7 \\
\hline
\end{tabular}

According to a study by Agarwal et al., 2008 India Chlamydia pneumoniae has been discussed as a possible co factor causing chronic obstructive pulmonary disease (COPD) and asthma (Agarwal, 2008). They detected IgM antibodies for Chlamydia 
pneumoniae in $18.3 \%$ of patients. in contrast to above study our study detected only $4 \%$ of chlamydia pneumonia by ELISA. According to Miyashita et al., (1998) and Gencay et al., (2001), the rate of 3\% compares favourably to serological studies of Chlaymdia pneumoniae in COPD and asthma (Miyashita, 1998 and Gencay, 2001).

According to study by Nagesh et al., (2004) found that Serological testing is considered the most useful means of determining the prevalence of $C$. pneumoniae infection MIF is currently the standard in $C$. pneumoniae serology, but is subjective and requires an expert microscopist to interpret the results. Inter-laboratory variation of MIF shows an overall agreement with reference standard titres of c. $80 \%$. In comparison, ELISA is more objective than MIF. It can be automated, with the advantages of high throughput and electronic records. ELISA is therefore easier to standardise and is the preferred diagnostic method ELISA may become a preferred objective test in the sero epidemiological study of $C$. pneumoniae infection and its link with atherosclerotic vascular disease (Nagesh, 2004).

According to study by Surinder Kumar et al., 2011 India tachypnea was documented in 9 (75\%) cases; cough and coryza in $12(100 \%)$ cases; fever in $8(66.67 \%)$, while $4(33.33 \%)$ cases were a febrile (Kumar, 2011).

Five (41.67\%) cases documented audible/auscutable wheezing and 3 (25\%) crepitations. The presence of $C$. pneumoniae antibody was higher in $10(7.87 \%)$ males than in $2(2.74 \%)$ females.

Our study detected chlamydia pneumonia antibodies in 5 male patients and none in the female patients.

Urinary antigen detection has been treated as the most specific reference test for diagnosis of legionellosis, this test has not been utilized in our study due to cost effective.

\section{References}

Agarwal A, Chander Y. Chronic Chlamydia pneumoniae infection and bronchial asthma: Is there a link? Indian J Med Microbiol 2008; 26:338-41.

Almudena Rojas, M. Dolores Navarro, Francisca E. Forne's, Estefanı'a Serra, Encarnación Simarro, Jose' Rojas, and Joaqu'n Ruiz Value of Serological Testing for Diagnosis of Legionellosis in Outbreak Patients. Journal of Clinical Microbiology, Aug. 2005, vol. 43(8): 4022-4025.

Chanock RM, Hayflick L, Barile MF. Growth on artificial medium of an agent associated with atypical pneumonia and its identification as a PPLO. Proc Natl Acad Sci U S A 1962; 48:41.

Cao B, Ren LL, Zhao F, Gonzalez R, Song SF, Bai L, et al. Viral and Mycoplasma pneumoniae community-acquired pneumonia and novel clinical outcome evaluation in ambulatory adult patients in China. Eur J Clin Microbiol Infect Dis 2010; 29: 1443-8.

Diederen BM. Legionella spp. and Legionnaires' disease. J Infect Dis; 2008; 56: $1-12$.

Cloud JL, Carroll KC, Pixton P, Erali M, Hillyard DR. Detection of Legionella species in respiratory specimens using PCR with sequencing confirmation. $J$ Clin Microbiol. 2000; 38(5): 1709-1712.

Liu, Fang-Ching, Chen Po-Yen, Huang Fang, Tsai Chi-Ren, Lee Chun-Yi, Lin Chen-Fu. (2008). Do serological tests provide adequate rapid diagnosis of Mycoplasma pneumoniae infection? Japanese journal of infectious diseases. 61. 397-9.

Grayston JT, Campbell LA, Kuo CC, Mordhorst CH, Saikku P, Thom DH, et al. A new respiratory tract pathogen: Chlamydia pneumoniae strain TWAR. J Infect Dis 1990; 161: 618.

Gencay M, Rudiger JJ, Tamm M, Soler M, 
Perruchoud AP, Roth M. Increased frequency of Chlamydia pneumoniae antibodies in patients with asthma. Am J Respir Crit Care Med 2001; 163: 10971100.

Huang HH, Zhang YY, Huang SG, Lu Q, Zhou $\mathrm{X}, \mathrm{Xiu} \mathrm{QY}$, et al. Investigation on etiology of community-acquired pneumonia in Shanghai. Chin J Infect Chemother., 2003; 3.

J. Ngeh, S. Gupta and C. Goodbourn. The reproducibility of an enzyme-linked immunosorbent assay for detection of Chlamydia pneumoniae-specific antibodies Clin Microbiol Infect 2004; 10: 171-174: 321-4. 01; 25.

Kumar S, Saigal SR, Sethi GR. Detection of IgM and IgG antibodies to Chlamydophila pneumoniae in pediatric communityacquired lower respiratory tract infections Indian J Pathol Microbiol 2011: 54(4): 7825.

Liu Fang-Ching, Chen Po-Yen, Huang Fang, Tsai Chi-Ren, Lee Chun-Yi and Lin, Chen$\mathrm{Fu}$. (2008). Do serological tests provide adequate rapid diagnosis of Mycoplasma pneumoniae infection? Japanese journal of infectious diseases. 61. 397-9.

Liu YN, Chen MJ, Zhao TM, Wang H, Wang $\mathrm{R}$, Liu QF, et al. A multicentre study on the pathogenic agents in 665 adult patients with community-acquired pneumonia in cities of China. Zhonghua Jie He He Hu Xi Za Zhi 2006; 29: 3-8.

Monsieur I, Meysman M, Vincken W, Huyghens L, Lauwers S. Severe community-acquired pneumonia caused by atypical organisms. Acta Clin Belg 1997; 52: 112-5.
M. W. Carter, S. A. H. Al-Mahdawi, I. G. Giles, J. D. Treharne, M. E. Ward and I. N. Clarke Nucleotide sequence and taxonomic value of the major outer membrane protein gene of Chlamydia pneumoniae IOL-207 Journal of General Microbiology (1991), 137, 465475.

Porath A, Schlaeffer F, Lieberman D. The epidemiology of community-acquired pneumonia among hospitalized adults. J Infect 1997; 34: 41-8.

Qu JX, Gu L, Wu J, Li XL, Dong JP, Pu ZH, et al. Epidemiological analysis of Mycoplasma pneumoniae acute infection in adults with community-acquired pneumonia. Zhonghua Liu Xing Bing Xue ZaZhi 2012; 33: 545-6.

Rama Chaudhry, Sutikshan Sharma, Sabah Javed, Kapil Passi, A.B. Dey and Pawan Malhotra Molecular detection of Mycoplasma pneumoniae by quantitative real-time PCR in patients with community acquired pneumonia Indian J Med Res, August 2013; 138(2): 244-51

Sabah Javed, Rama Chaudhry, Kapil Passi, Sutikshan Sharma, Padmaja K., Benu Dhawan and A.B. Dey Sero diagnosis of Legionella infection in community acquired Pneumonia Indian J Med Res, January 2010: 131; 92-96.

Ewing, S. Legionella spp. in acute exacerbations of chronic obstructive pulmonary disease: what is the evidence? Eur Respir J 2002; 19: 387-389

Yu RH, Zang GQ, Liu G, Le WL. Etiology and clinical features of 89 patients with leukopenia or normal leucocyte community acquired pneumonia. Chin $\mathrm{J}$ Clin Med 2003; 3: 321-4.

\section{How to cite this article:}

Sowjanya, G., Amar C. Sajjan, G. Swetha, B. Archana and Aparna. 2019. Prevalence of Mycoplasma pneumoniae, Chlamydia pneumoniae and Legionella pneumophila in LRTI Patients in a Tertiary Care Center, Karimnagar. Int.J.Curr.Microbiol.App.Sci. 8(05): 15511556. doi: https://doi.org/10.20546/ijcmas.2019.805.179 